\title{
On the maximum efficiency of the propeller mass-ejection mechanism
}

\author{
M. Falanga ${ }^{1}$, E. Bozzo ${ }^{2,3}$, L. Stella ${ }^{2}$, L. Burderi ${ }^{4}$, T. Di Salvo ${ }^{5}$, and R. Perna ${ }^{6}$ \\ ${ }^{1}$ CEA Saclay, DSM/DAPNIA/Service d'Astrophysique (CNRS FRE 2591), 91191 Gif-sur-Yvette, France \\ e-mail: mfalanga@cea.fr \\ 2 INAF - Osservatorio Astronomico di Roma, via Frascati 33, 00044 Rome, Italy \\ 3 Dipartimento di Fisica - Università di Roma "Tor Vergata", via della Ricerca Scientifica 1, 00133 Rome, Italy \\ 4 Università degli studi di Cagliari, Dipartimento di Fisica, SP Monserrato-Sestu, KM 0.7, 09042 Monserrato, Italy \\ 5 Department of Astrophysical and Planetary Sciences, University of Colorado, Boulder, CO 80309, USA
}

Received 11 January 2005 / Accepted 11 January 2007

\begin{abstract}
Aims. We derive simple estimates of the maximum efficiency with which matter can be ejected by the propeller mechanism in diskfed, rotating magnetic neutron stars. Some binary evolution scenarios envisage that this mechanism is responsible for expelling to infinity the mass inflowing at a low rate from the companion star, therefore limiting the total amount of mass that can be accreted by the neutron star.

Methods. We demonstrate that, for typical neutron star parameters, a maximum of $\eta_{\text {pro }}<5.7\left(P_{-3}\right)^{1 / 3}$ times more matter than accreted can be expelled through the propeller mechanism at the expenses of the neutron star rotational energy $\left(P_{-3}\right.$ is the NS spin period in unit of $10^{-3} \mathrm{~s}$ ). Approaching this value, however, would require a great deal of fine tuning in the system parameters and the properties of the interaction of matter and magnetic field at the magnetospheric boundary.

Results. We conclude that some other mechanism must be invoked in order to prevent that too much mass accretes onto the neutron stars of some low mass X-ray binaries.
\end{abstract}

Key words. accretion, accretion: disks - stars: binaries: close - stars: neutron - stars: pulsars: general

\section{Introduction}

One of the possible endpoints of the evolution of the compact object in a low-mass X-ray binary (LMXB) is a millisecond pulsar (MSP), a rapidly spinning neutron star (NS) with weak surface magnetic field $\left(\sim 10^{8}-10^{9} \mathrm{G}\right)$. Such NSs are thought to be spun-up to millisecond periods by the accretion of mass and angular momentum from matter in a disk formed through Rochelobe overflow of a low-mass companion star (for a review see e.g., Bhattacharya \& van den Heuvel 1991). After the accretion phase has ended, the NS may turn on as a radio MSP. In recent years, the prediction that LMXB host NSs with spin periods in the ms range has been confirmed through the discovery of 300-600 Hz nearly-coherent oscillations during type I X-ray bursts from some 10 LMXBs of the Atoll class (e.g., Strohmayer et al. 1997). This was followed by the detection of coherent X-ray pulsations in the persistent emission of seven low luminosity transients of the same class. The spin frequencies lie between 180 and $600 \mathrm{~Hz}$ and the orbital periods between $40 \mathrm{~min}$ and $5 \mathrm{~h}$ (see review by Wijnands 2005). For the first time, the predicted decrease of the NS spin period during accretion was measured in the accreting MSP IGR J00291+5934 (Falanga et al. 2005; Burderi et al. 2006). This provided a strong confirmation of the theory of "recycled" pulsars in which the old NS in LMXBs become millisecond radio pulsars through spin-up by transfer of angular momentum by the accreting material.

All accreting MSPs are X-ray transient systems. They spend most of the time in a quiescent phase, with X-ray luminosities of the order of $10^{31}-10^{32} \mathrm{erg} \mathrm{s}^{-1}$, and occasionally show weeks to months long outbursts, reaching X-ray luminosities of
$10^{36}-10^{37} \mathrm{erg} \mathrm{s}^{-1}$, during which coherent X-ray pulsations are observed. The magnetic fields inferred for these NS are of order $10^{8}-10^{9}$ G (e.g., Campana et al. 1998; Psaltis \& Chakrabarty 1999; Di Salvo \& Burderi 2003). These findings added a great deal of confidence in the likely connection between accreting NS and millisecond radio pulsars.

Models for the evolution of LMXBs as progenitors of radio MSPs still involve significant assumptions about the transfer of mass from one star to the other and the role of dynamically influent NS magnetic fields (Podsiadlowski et al. 2002). The MSP formation depends on the amount of matter accreted from the companion which, in turn, is likely limited by mass ejection from the binary. Cook et al. (1994a), following a full GR treatment, show that a NS of $1.4 M_{\odot}$ has to accrete $\sim 0.2-0.5 M_{\odot}$ to reach periods in the range $0.6-1.5 \mathrm{~ms}$ (see also e.g., Burderi et al. 1999). Most donor stars in systems hosting recycled radio pulsars must have lost most of their mass during their evolution. They now appear as white or brown dwarfs of mass $\sim 0.15-0.30 M_{\odot}$ (Taam et al. 2000), the progenitors of which were likely stars of $\sim 1.0-2.0 M_{\odot}$ (e.g., Burderi et al. 1996; Tauris \& Savonije 1999). Hence, a crucial issue for evolutionary models for MSPs is which fraction of mass lost by the companion $\left(M_{\text {lost }} \sim 0.7-1.8 M_{\odot}\right)$ effectively accretes onto the NS. Mass ejection must be efficient, at least in some cases, to avoid that accretion of a large amount of mass onto the NS could induce collapse to a black hole. For most equations of state of ultra-dense matter, this is expected to occur for NS masses above $\sim 2 M_{\odot}($ Cook et al. 1994b). Different models have been proposed to explain how mass can be ejected from binary 
system, thus limiting the amount of matter that is accreted by the NS to about 0.2-0.4 $M_{\odot}$ (Illarinov \& Sunyaev 1975; Wang \& Robertson 1985; Romanova et al. 2005; Ustyugova et al. 2006).

In this work we use simple arguments to derive an upper limit on the efficiency of the propeller mass-ejection mechanism. In Sect. 2 we consider accretion and ejection of infinitesimally small amounts of matter, the case relevant to NS X-ray transient. Since in this case the NS parameters do not change significantly between each accretion/ejection cycle an analitic expression for the propeller efficiency can be derived. In Sect. 3 we extend this limit to the case of accretion and ejection of finite amounts of matter.

\section{Ejector-propeller efficiency}

Matter transferred from the companion star via Roche-Lobe overflow possesses enough specific angular momentum that an accretion disk forms around the accreting NS. The disk is truncated at the magnetospheric radius, $R_{\mathrm{m}}$. Different calculations, carried out by adopting different models and/or assumptions, produce only slightly different estimates of the value of $R_{\mathrm{m}}$ (a factor of 2-3 at the most, e.g., Rappaport \& Joss 1977; Ghosh \& Lamb 1979; Wang 1987).

The value of $R_{\mathrm{m}}$ as a function of the system parameters is only of marginal relevance to the present paper. Therefore we consider here the value inferred from simple theory (see e.g., Eq. (4) of Lamb \& Pethick 1974); that is

$R_{\mathrm{m}}=3.2 \times 10^{8} M_{1}^{-1 / 7} \mu_{30}^{4 / 7} \dot{M}_{17}^{-2 / 7} \mathrm{~cm}$,

where $M_{1}$ is the NS mass in units of $1 M_{\odot}, \mu_{30}$ is the NS magnetic dipole moment in units of $10^{30} \mathrm{G} \mathrm{cm}^{3}$, and $\dot{M}_{17}$ is the mass accretion rate in units of $10^{17} \mathrm{~g} \mathrm{~s}^{-1}$.

Disk accretion onto a spinning, magnetic NS is expected to be centrifugally inhibited, once the magnetospheric radius is larger than the corotation radius, $R_{\text {co }}$, and inside the light cylinder radius, $R_{\mathrm{lc}}$ (Illarinov \& Sunyaev 1975). The corotation radius, $R_{\text {co }}$, is the radius at which the Keplerian frequency of the orbiting matter is equal to the NS spin frequency: $R_{\mathrm{co}}=$ $\left(G M / \Omega_{0}^{2}\right)^{1 / 3}=1.5 \times 10^{6} P_{-3}^{2 / 3}\left(M / M_{\odot}\right)^{1 / 3} \mathrm{~cm}$, where $\Omega_{0}$ is the angular velocity and $P_{-3}$ is the spin period of the NS in milliseconds. The light cylinder radius, $R_{\mathrm{lc}}$, is the radius at which the corotating magnetic field lines reach the speed of light $c$, $R_{\mathrm{lc}}=c / \Omega_{0}$. Outside $R_{\mathrm{lc}}$ the magnetic field lines cannot corotate any longer and a radiative electromagnetic field must be generated. If an element of matter $\mathrm{d} M_{\text {in }}$ is accreted onto a NS from a Keplerian disk, part of its energy is stored as rotational energy of the NS; this can later be used to eject a mass $\mathrm{d} M_{\text {out }}$. We define the propeller efficiency as the ratio of the ejected to accreted matter $\eta_{\text {pro }} \equiv \mathrm{d} M_{\text {out }} / \mathrm{d} M_{\text {in }}$. Moreover we assume that only the rotational energy gained by the star during the previous accretion phase can be used to eject matter in the propeller phase.

In order to determine the rotational energy gained by the NS we first consider the accretion phase. During accretion the rate of rotational energy transferred to the NS can be written in terms of the transfer of angular momentum, $L$, at the inner boundary of the disk:

$\dot{E}_{\mathrm{rot}}=\Omega_{0} \dot{L}-\frac{1}{2} \Omega_{0}^{2} \dot{I}$

where $\dot{L}=I \dot{\Omega}_{0}+\Omega_{0} \dot{I}=\dot{M} l$. Here $I=\beta^{-1} M R_{\mathrm{NS}}^{2}$ is the moment of inertia of the NS, and $l$ is the specific angular momentum of the accreted mass. For NS models with mass above an initial value of $M \sim 1.4 M_{\odot}$ (Weaver et al. 1978) and well below the maximum mass, values of $\beta$ are expected in the 2.3-3.1 range for a variety of equations of state. In our calculations we use $\beta=2.5$, the value for a uniform Newtonian star. For a disk truncated at the magnetospheric radius, $R_{\mathrm{m}}$, we approximate the specific angular momentum transferred to the NS with the Keplerian value at $R_{\mathrm{m}}$, i.e. $l \simeq l_{\mathrm{m}}=\left(G M R_{\mathrm{m}}\right)^{1 / 2}$. According to detailed models of the disk-magnetosphere interaction this approximation is accurate in the limit in which $R_{\mathrm{m}} \ll R_{\text {co }}$, whereas it provides an upper limit in the case in which $R_{\mathrm{m}} \simeq R_{\mathrm{co}}$ and non-material (spin-down) torques due to the interaction of the NS magnetic field with disk regions outside $R_{\mathrm{co}}$, become important (Ghosh \& Lamb 1979; Wang 1987; Rappaport et al. 2004). Since our aim here is to derive an upper limit on the propeller efficiency, we can safely adopt the Keplerian value of the specific angular momentum. Equation (2) thus writes

$\mathrm{d} E_{\mathrm{rot}}=\Omega_{0}\left(G M R_{\mathrm{m}}\right)^{1 / 2} \mathrm{~d} M_{\mathrm{in}}-\frac{1}{2} \beta^{-1} R_{\mathrm{NS}}^{2} \Omega_{0}^{2} \mathrm{~d} M_{\mathrm{in}}$,

which describes the amount of energy accreted from a disk ending at the magnetospheric radius. For a given angular velocity of the NS, the rotational energy, $\mathrm{d} E_{\text {rot }}$, is highest when the magnetospheric radius, $R_{\mathrm{m}}$, takes the largest value, compatible with accretion onto the NS surface: that is at $R_{\mathrm{m}}=R_{\mathrm{co}}$. Setting $R_{\mathrm{m}}=R_{\mathrm{co}}$ Eq. (3) gives

$\mathrm{d} E_{\text {rot }}=\frac{G M}{R_{\text {co }}} \mathrm{d} M_{\text {in }}-\frac{1}{2} \beta^{-1} R_{\mathrm{NS}}^{2} \Omega_{0}^{2} \mathrm{~d} M_{\text {in }}$.

This is the maximum increase in $E_{\text {rot }}$ that an element of diskaccreting mass $\mathrm{d} M_{\text {in }}$ can cause.

We now consider the propeller phase. According to the virial theorem, the energy required to eject a disk mass element, $\mathrm{d} M_{\text {out }}$, from a radius $R$ to infinity, is $\mathrm{d} E_{\text {eje }}=G M \mathrm{~d} M_{\text {out }} / 2 R$. In order to estimate the maximum possible propeller efficiency we suppose that ejection takes place from the largest possible radius at which the propeller can work, such that the energy required to eject is minimized. That is $R=R_{\mathrm{lc}}$.

Setting $\mathrm{d} E_{\mathrm{rot}}=\mathrm{d} E_{\text {eje }}$ and assuming that ejection takes place at $R_{\mathrm{lc}}$, we have for the maximum efficiency of the propeller:

$\eta_{\text {pro }} \leq 6.37\left(\frac{M}{M_{\odot}}\right)^{-1 / 3}\left(P_{-3}\right)^{1 / 3}-0.57\left(\frac{M}{M_{\odot}}\right)^{-1}\left(P_{-3}\right)^{-1}$

where we used $R_{\mathrm{NS}}=10 \mathrm{~km}$.

An alternative possibility is that there is no magnetosphere in the accretion phase, and the disk extends all the way to the NS surface (such that we set formally $R_{\mathrm{m}} \simeq R_{\mathrm{NS}}$, where $R_{\mathrm{NS}}$ is the NS radius). This might be the case in LMXBs which do not display coherent pulsations in their persistent X-ray flux. In this case

$$
\begin{aligned}
\eta_{\text {pro }} & =\frac{\Omega_{0}\left(G M R_{\mathrm{NS}}\right)^{1 / 2}}{G M / 2 R_{\mathrm{lc}}}-\frac{\beta^{-1} R_{\mathrm{NS}}^{2} \Omega_{0}^{2}}{G M / R_{\mathrm{lc}}} \\
& =2\left(\frac{R_{\mathrm{NS}}}{R_{\mathrm{g}}}\right)^{1 / 2}-\frac{c \beta^{-1}}{G M} R_{\mathrm{NS}}^{2} \Omega_{0},
\end{aligned}
$$

with $R_{\mathrm{g}}=G M / c^{2}=1.48 \times 10^{5}\left(M / M_{\odot}\right) \mathrm{cm}$ the gravitational radius.

For a typical NS, $R_{\mathrm{NS}} \simeq 6 R_{\mathrm{g}}$, one has

$\eta_{\text {pro }} \leq 5.20\left(\frac{M}{M_{\odot}}\right)^{-1 / 2}-0.57\left(\frac{M}{M_{\odot}}\right)^{-1}\left(P_{-3}\right)^{-1}$

a result that is similar to that in Eq. (5). 

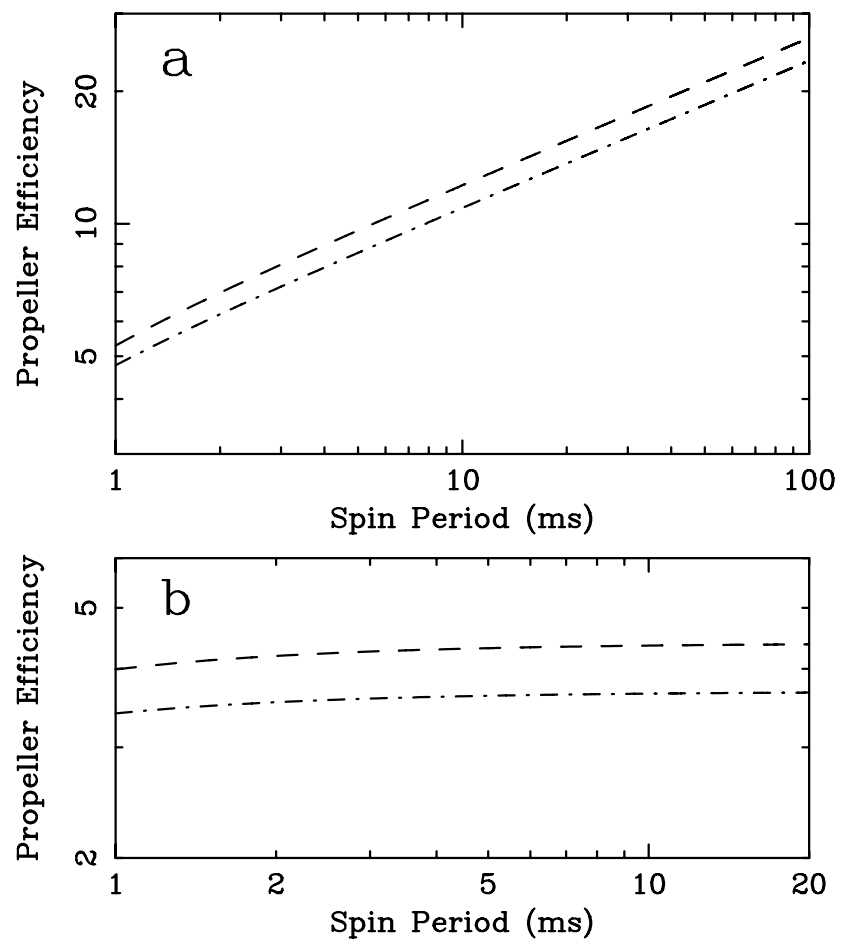

Fig. 1. The highest possible propeller efficiency, $\eta_{\text {pro }}$, the ratio of the ejected mass to the accreted mass, versus spin period. The dashed and dot-dashed lines correspond to Eq. (5) (panel a) and Eq. (7) (panel b), for a NS of $1.4 M_{\odot}$ and $2.0 M_{\odot}$, respectively.

The above derived limits on $\eta_{\text {pro }}=\mathrm{d} M_{\text {out }} / \mathrm{d} M_{\text {in }}$ involve a great deal of fine tuning with respect to the ejection of matter from close to the light cylinder. Indeed, despite the fact that the NS magnetic field lines approach $c$ at $R_{\mathrm{lc}}$, it assumed that ejection takes place at much lower speeds, precisely the lowest required for matter to reach infinity.

Equations (5) and (7) are plotted in Fig. 1 as a function of the spin period and for different values of the NS mass. In the following we discuss the impact of the limits in Eqs. (5) and (7) on evolutionary models of accreting NS's binaries and MSP's.

\section{Discussion}

The limits that we have derived on the mass ejection efficiency of the propeller mechanism are based on the assumption that, when accretion takes place, the highest angular momentum is transferred to the NS, whereas ejection occurs at the expense of the NS rotational energy from the largest radius compatible with the propeller mechanism. This minimizes the loss of rotational energy.

Somewhat different limits are obtained depending on whether during the accretion phase, the disk ends at the magnetospheric boundary or extends all the way to the NS surface. The latter case might be relevant to high luminosity NS systems which do not display periodic pulsations. The corresponding limit on $\eta_{\text {pro }}$ (see Eq. (7)) applies only over a limited range of fast NS spin periods. Indeed, if the magnetosphere is "squeezed" inside the star surface during the accretion phase (formally $R_{m} \simeq R_{\mathrm{NS}}$ ), then, as the mass inflow rate decreases by up to $\sim 4-5$ orders of magnitude (as inferred from the luminosity NS X-ray transient systems, e.g., Campana \& Stella 2000), the magnetosphere can expand up to $\sim 20 R_{\mathrm{NS}}$ at the maximum. This is due to the stiffness of the NS magnetosphere, reflected in the weak dependence of $R_{\mathrm{m}}$ on $\dot{M}$ in Eq. (1). For the NS to be in propeller phase the corotation radius, $R_{\mathrm{co}}$, must be smaller than the magnetospheric radius $R_{\mathrm{m}}\left(\sim 20 R_{\mathrm{NS}}\right)$, in turn implying that the NS spin cannot be longer than $\sim 30 \mathrm{~ms}$ (for NS with spin period longer than this, the propeller efficiency limit in Eq. (7) would thus be inapplicable). In this case, from Fig. 1 (panel b), we note that the maximum propeller efficiency cannot be larger than $\simeq 4$ for NS with a spin period of $\simeq 1 \mathrm{~ms}$. The limit on $\eta_{\text {pro }}$ derived in the case in which there exists a magnetosphere also during the accretion phase (see Eq. (5)) applies instead over a large range of spin periods and its value is $\sim 5$, for a spin period of $\simeq 1 \mathrm{~ms}$ and increases for longer periods.

Strictly speaking, our reasoning applies only to the accretion/ejection of (infinitesimally) small amounts of matter. The limits we derived are thus immediately relevant to NSs that often cycle between the accretion and ejection regimes. These include transient X-ray systems which alternate active periods, lasting up to years, to quiescent intervals of up to $\sim 100 \mathrm{yr}$. During each outburst cycle the NS paramaters (e.g., mass, spin period, magnetic dipole moment) change only by very small amounts. These systems likely evolve through a very long sequence of accretion/ejection cycles, while within each cycle the efficiency of the propeller ejection remains limited by the values we have derived. It is easy to see that in this case the overall efficiency must be limited by the maximum possible value of $\eta_{\text {pro }}$, given by Eq. (5) independent of whether accretion occurs through the magnetosphere or not.

Therefore, flunging away matter with higher efficiency than permitted by the above limit would require a different ejection mechanism such as the radio pulsar ejection mechanism (Illarinov \& Sunyaev 1975). This mechanism can operate at much larger radii (comparable to the NS Roche-lobe radius) where the gravitational potential is much shallower and thus the energy required to expel matter to infinity is much smaller (e.g., Burderi et al. 2001).

In the case of accretion and ejection of finite amounts of matter, an analitical expression of $\eta_{\text {pro }}$ cannot be found. In fact, in this case, one has to consider the variation of the stellar parameters during the evolution of the system. The equations relating the rotational energy acquired during accretion to the ejection energy lost during the propeller phase depend on the instantaneous value of the stellar mass and angular velocity, and their evolution can be followed numerically. To obtain an estimate of the maximum propeller efficiency we again assumed that the accretion phase takes place from the corotation radius, the ejection phase from $R_{\mathrm{lc}}$ and that all the rotational energy acquired during accretion is used to eject matter from the system. In our numerical integrations we neglected the decrease in the NS radius which results from increasing its mass through accretion. In any case, using the simple relation $R_{\mathrm{NS}} \propto M_{\mathrm{NS}}^{-1 / 3}$ we checked that this is a small effect and the induced variations on $\eta_{\text {pro }}$ are less that $\sim 2 \%$. The parameters that we used in our calculations are those in the evolutionary models of Ergma \& Sarna (2003) and Tauris $\&$ Savonije (1999). In the first paper the authors assume that propeller ejection takes place from an increasing radius, as the mass inflow rate toward the NS decreases. Using their parameters (Ergma \& Sarna 2003), i.e. NS mass of $\sim 1.4 M_{\odot}$, initial spin period $\sim 0.03 \mathrm{~s}, \sim 0.25 M_{\odot}$ accreted onto the NS, and a final spin period of $\sim 3.6 \mathrm{~ms}$, our calculations show that the efficiency is bound by the maximum value of $\lesssim 5$. This is a resonable result since accretion causes $\Omega_{0}$ to increase and consequently, ejection takes place (mainly) at smaller values of $R_{\mathrm{lc}}$. In this case the energy required to eject matter is higher and the propeller less efficient. An additional effect comes from the fact that the corotation 


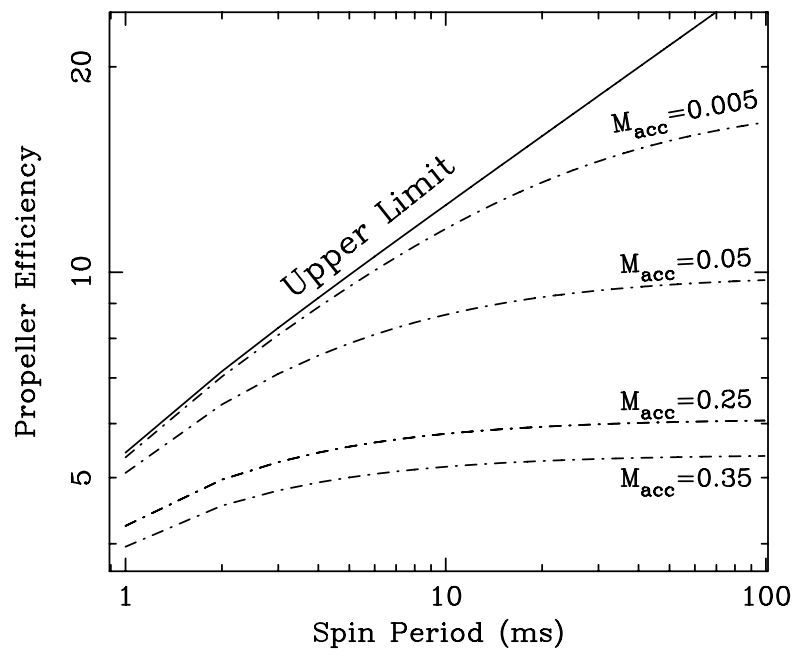

Fig. 2. The highest possible propeller efficiency, $\eta_{\text {pro }}$, versus spin period for different amounts of mass accreted onto a $1.3 M_{\odot}$ NS are shown by dot-dashed lines. The solid line is the propeller efficiency upper limit Eq. (5).

radius slightly increases during accretion due to the building up of the NS mass. However, our calculations show that this effect is small (due to the limited range in NS mass). Infact in all cases we obtained a lower efficiency than the maximum value of $\eta_{\text {pro }}$ of Eq. (5), which holds for short accretion/ejection cycles. Figure 2 shows that the upper limit of Eq. (5) is approached only when the accreted mass on the NS is very small. We note that the propeller efficiency required in the work of Ergma \& Sarna (2003) is $\sim 2.6$, consistent with the limit we have derived. It is more difficult to carry out a comparison with the work of Tauris \& Savonije (1999). We refer to their models for relatively close binary systems with light donors $\left(P_{\text {orb }}<10 \mathrm{~d}, M_{\text {donor }}<1.3 M_{\odot}\right)$. This is because, in those cases, the mass-transfer rate remains sub-Eddington during the entire binary system evolution, preventing the formation of super-Eddington strong outflows and causing the binary system to eject matter mainly via the propeller effect. We use a NS mass of $\sim 1.3 M_{\odot}, \sim 0.1-0.4 M_{\odot}$ accreted onto the NS and an initial NS spin period in the range $\sim 0.001-0.006 \mathrm{~s}$. Again we find the required propeller efficiency in Tauris \& Savonije (in some cases $\eta_{\text {pro }} \lesssim 1$ ) is smaller than our numerically estimated upper limit $\left(\eta_{\text {pro }} \lesssim 7\right)$.

A refined treatment of the propeller effect for a magnetic accreting ms pulsar was carried out by Ustyugova et al. (2006). Using a magnetohydrodynamic approach, and considering a detailed model for the interaction between the NS magnetosphere and the accretion disk, they showed that propeller outflows can be expected from a fast rotating $\left(P_{\text {spin }} \sim 1.2-1.6 \mathrm{~ms}\right)$ weakly magnetic NS $\left(B \sim 5 \times 10^{8}-2 \times 10^{9} \mathrm{G}\right)$. A comparison between their results and ours $\eta_{\text {pro }}$ is straightforward if one consider that all the NS rotational energy used in the propeller phase derives from accretion of matter at the corotation radius. Dividing their total ejected matter $\left(\dot{M}_{j}+\dot{M}_{w}\right)$ by the above estimated accreted matter, we obtain $\eta_{\text {pro }} \sim 0.4$, for a $1.26 \mathrm{~ms}$ pulsar with $B \sim 10^{9} \mathrm{G}$.

In summary the evolutionary calculation of Ergma \& Sarna (2003) and Tauris \& Savonije (1999) correspond to a propeller efficiency of $\sim 1-3$, which as such would be adequate to explain how the ejection of a large fraction of the mass transferred by the companion can take place in these systems. The upper limit that we have derived in this paper for the propeller efficiency is only a factor of $\sim 2$ higher. However achieving such a limit would require a high degree of fine tuning. This indicates that a propeller efficiency of order $\sim 2-3$ might be difficult to achieve in real systems.

As suggested by the deteiled propeller simulations by Ustyugova et al. (2006) a propeller efficiency $<1$ might be more realistic. If this was the case, then a different mechanism to eject from the binary most of the mass transferred by the companion is required. The onset of the radio pulsar ejection mechanism is thus probably to be favored.

Acknowledgements. M.F. acknowledges the French Space Agency (CNES). E.B. acknowledges support from ASI (I/023/05/0) and from the Italian Minister of University and Technological Research through grant PRIN2004023189. E.B. thanks G. Lavagetto for usefull discussions and CEA Saclay, DSM/DAPNIA/SAp for hospitality during part of this work.

\section{References}

Bhattacharya, D., \& van den Heuvel, E. P. J., 1991, Phys. Rep., 203, 1 Burderi, L., King, A. R., \& Wynn, G. A. 1996, ApJ, 457, 348

Burderi, L., Possenti, A., Colpi., M., Di Salvo, T., \& D’amico, N. 1999, ApJ, 519, 285

Burderi, L., Possenti, A., D’Antona, F., et al. 2001 ApJ, 560, L71

Burderi, L., Di Salvo, T., Lavagetto, G., et al. 2001, ApJ, in press [arXiv:astro-ph/0611222]

Campana, S., \& Stella, L. 2000, ApJ, 541, 849

Campana S., Colpi, M., Mereghetti, S., Stella, L., \& Tavani, M. 1998, A\&ARv, 8,279

Cook, G. B., Shapiro, S. L., \& Teukolski, S. A. 1994a, ApJ, 424, 823

Cook, G. B., Shapiro, S. L., \& Teukolski, S. A. 1994b, ApJ, 432, L117

Di Salvo, T., \& Burderi, L. 2003, A\&A, 397, 723

Ergma, E., \& Sarna, M. J. 2003, A\&A, 399, 237

Falanga, M., Kuiper, L., Poutanen, J., et al. 2005, A\&A, 444, 15

Ghosh, P., \& Lamb, F. K. 1979, ApJ, 234, 296

Illarinov, A., \& Sunyaev, R. 1975, A\&A, 39, 185

Lamb, F. K., \& Pethick, C. J. 1974, in Astrophysics and Gravitation, Proc. 16th Solvay Congress, 135

Podsiadlowski, P., Rappaport, S., \& Pfahl, E. D. 2002, ApJ, 565, 1107

Psaltis, D., \& Chakrabarty, D. 1999, ApJ, 521, 332

Rappaport, S., \& Joss, P. C. 1977, Nature, 266, 683

Rappaport, S. A., Fregeau, J. M., \& Spruit, H. 2004, ApJ, 606, 436

Romanova, M. M., Ustyugova, G. V., Koldoba, A. V., \& Lovelace, R. V. E. 2005, ApJ, 635, L165

Strohmayer, T. E., Zhang, W., \& Swank, J. H. 1997, ApJ, 487, L77

Taam, R. E., King, A. R., \& Ritter, H. 2000, ApJ, 541, 329

Tauris, T. M., \& Savonije, G. J. 1999, A\&A, 350, 928

Ustyugova, G. V., Koldoba, A. V., Romanova, M. M., et al. ApJ, 646, 304

Weaver, T. A., Zimmerman, G. B., \& Woosley, S. E. 1978, ApJ, 225, 1021

Wang, Y.-M. 1987, A\&A, 183, 257

Wang, Y. -M., \& Robertson, J. A. 1985, A\&A, 151, 361

Wijnands, R. 2005, in Pulsars New Research (NY: Nova Science Publishers), in press [arXiv:astro-ph/0501264] 